Portland State University

PDXScholar

High-Order Method for Evaluating Derivatives of Harmonic Functions in Planar Domains

Jeffrey S. Ovall

Portland State University, jovall@pdx.edu

Samuel E. Reynolds

Portland State University

Follow this and additional works at: https://pdxscholar.library.pdx.edu/mth_fac

Part of the Harmonic Analysis and Representation Commons

Let us know how access to this document benefits you.

Citation Details

Ovall, J. S., \& Reynolds, S. E. (2018). A High-Order Method for Evaluating Derivatives of Harmonic Functions in Planar Domains. SIAM Journal on Scientific Computing, 40(3), A1915-A1935.

This Post-Print is brought to you for free and open access. It has been accepted for inclusion in Mathematics and Statistics Faculty Publications and Presentations by an authorized administrator of PDXScholar. Please contact us if we can make this document more accessible: pdxscholar@pdx.edu. 


\title{
A HIGH-ORDER METHOD FOR EVALUATING DERIVATIVES OF HARMONIC FUNCTIONS IN PLANAR DOMAINS
}

\author{
JEFFREY S. OVALL AND SAMUEL E. REYNOLDS
}

\begin{abstract}
We propose a high-order integral equation based method for evaluating interior and boundary derivatives of harmonic functions in planar domains that are specified by their Dirichlet data.
\end{abstract}

\section{INTRODUCTION}

Let $\Omega \subset \mathbb{R}^{2}$ be a bounded, open and simply connected domain, whose boundary $\partial \Omega$ is either smooth or consists of a finite number of smooth closed arcs that intersect only at corners having interior angles strictly between 0 and $2 \pi$ (no slits or cusps). Given data $f \in C(\partial \Omega)$ that is also smooth or piecewise smooth, we consider the Dirichlet problem

$$
\Delta u=0 \text { in } \Omega, u=f \text { on } \partial \Omega .
$$

This paper concerns the efficient and accurate approximation of derivatives of $u$ at points in $\Omega$, as well as the normal derivative $\partial u / \partial \mathbf{n}$ at points on $\partial \Omega$ (the Dirichlet-to-Neumann map $f \mapsto \partial u / \partial \mathbf{n}$ ), by doing computations only on $\partial \Omega$. Throughout, $\mathbf{n}$ and $\mathbf{t}$ (where they are defined) are, respectively, the outward unit normal vector and the unit tangent vector in the counter-clockwise direction on $\partial \Omega$.

A motivation for developing an efficient and high-order solver for (1) having these features comes from Boundary Element-Based Finite Element Methods (BEM-FEM) (cf. $[2,5,6,13,14,17-19]$ ), an approach to solving boundary value problems on fairly general polygonal or polyhedral partitions (meshes) of the given domain. BEM-FEM employs local spaces of functions that are defined implicitly on each mesh cell in terms of boundary value problems - typically Poisson problems having polynomial righthand side and Dirichlet data, which can be reduced to computing harmonic functions with prescribed (polynomial) Dirichlet data. Functions in these local spaces, together with their interior derivatives (at least gradients) and their boundary normal derivatives, are evaluated in the formation of the associated finite element linear systems. We propose an integral equation based approach that efficiently provides high-quality approximations of each of these quantities of interest, also allowing for more general cell shapes and boundary conditions in a natural way.

Two key ingredients of the proposed approach are Cauchy's integral formulas and the notion of harmonic conjugates. Recall that functions $u, v$ are called harmonic conjugates in $\Omega$ when $\Delta u=\Delta v=0$ in $\Omega$, and they satisfy the Cauchy-Riemann equations

$$
\nabla u=R \nabla v \text { in } \Omega, \text { where } R=\left(\begin{array}{cc}
0 & 1 \\
-1 & 0
\end{array}\right) .
$$

In other words, the gradients of $u$ and $v$ are orthogonal in $\Omega$, with $\nabla u$ being a clockwise rotation of $\nabla v$ by $\pi / 2$. Given $u$ that is harmonic in $\Omega$, it is guaranteed a family of harmonic conjugates that only differ by additive constants. We identify $\Omega$ with a subset of $\mathbb{C}$ in the natural way via $z=x_{1}+\mathfrak{i} x_{2}$ for $x=\left(x_{1}, x_{2}\right) \in \Omega$. Given a harmonic conjugate $v$ of $u$, the function $w(z)=u(x)+\mathfrak{i} v(x)$ is analytic in $\Omega$, and we have the Cauchy integral formulas

$$
w^{(k)}\left(z_{0}\right)=\frac{k !}{2 \pi \mathfrak{i}} \int_{\partial \Omega} \frac{w(z)}{\left(z-z_{0}\right)^{k+1}} d z
$$

Date: August 2, 2017.

Ovall was partially supported by the NSF Grant DMS-1414365. 
From (3) we can extract values and derivatives of $u$ (and $v$ ) at $x_{0}=\left(x_{0,1}, x_{0,2}\right)$ by looking at the real and imaginary parts of this integral. For example,

$$
\begin{aligned}
w^{\prime}\left(z_{0}\right) & =u_{x_{1}}\left(x_{0}\right)-\mathfrak{i} u_{x_{2}}\left(x_{0}\right)=v_{x_{2}}\left(x_{0}\right)+\mathfrak{i} v_{x_{1}}\left(x_{0}\right), \\
w^{\prime \prime}\left(z_{0}\right) & =u_{x_{1} x_{1}}\left(x_{0}\right)-\mathfrak{i} u_{x_{1} x_{2}}\left(x_{0}\right)=v_{x_{1} x_{2}}\left(x_{0}\right)+\mathfrak{i} v_{x_{1} x_{1}}\left(x_{0}\right) .
\end{aligned}
$$

The remaining second partials of $u$ and $v$ are clear from the fact that they are harmonic.

The orthogonality of the gradients of $u$ and $v$ imply that

$$
\frac{\partial u}{\partial \mathbf{n}}=\frac{\partial v}{\partial \mathbf{t}} \quad, \quad \frac{\partial v}{\partial \mathbf{n}}=-\frac{\partial u}{\partial \mathbf{t}}=-\frac{\partial f}{\partial \mathbf{t}} \text { on } \partial \Omega .
$$

Using the latter of these, a specific harmonic conjugate of $u$ is given as the solution of a complementary Neumann problem for (1),

$$
\Delta v=0 \text { in } \Omega \quad, \quad \frac{\partial v}{\partial \mathbf{n}}=g=-\frac{\partial f}{\partial \mathbf{t}} \text { on } \partial \Omega \quad, \quad \int_{\partial \Omega} v d S=0 .
$$

The final condition is a convenient way of selecting a specific harmonic conjugate. The proposed strategy for computing $\partial u / \partial \mathbf{n}$ on $\partial \Omega$, and derivatives of $u$ in $\Omega$, may be summarized as follows:

(a) Compute the solution of (7) on $\partial \Omega$ (a Neumann-to-Dirichlet map $g \mapsto v$ ). This is done in the present work by solving a second-kind integral equation via a Nyström method.

(b) Use $\partial v / \partial \mathbf{t}$ to compute $\partial u / \partial \mathbf{n}$ on $\partial \Omega$.

(c) Use (3) to compute derivatives of $u$ in $\Omega$.

Some of the key computational tools are quadratures based on the trapezoid rule, and the Fast Fourier Transform.

In Section 2 we describe the approach outlined above in the case of smooth boundaries and data, and numerically demonstrate the high-order nature of the algorithm. In Section 3, we do the same in the case of piecewise smooth boundaries and data, highlighting the challenges when corners are present, and how we deal with them.

\section{Smooth Boundary and Data}

We assume here that $\partial \Omega$ and $f$ are smooth (at least $C^{2}$ ). A direct application of Green's Theorem shows that the solution of $(7)$ satisfies

$$
v(x)=\int_{\partial \Omega} \Phi(x, y) g(y) d S(y)-\int_{\partial \Omega} \frac{\partial \Phi(x, y)}{\partial \mathbf{n}(y)} v(y) d S(y) \text { for } x \in \Omega,
$$

where $\Phi(x, y)=-(2 \pi)^{-1} \ln |x-y|$ is the fundamental solution of the Laplacian. From (8), we obtain the following second-kind integral equation for the Dirichlet trace of $v$,

$$
\frac{v(x)}{2}+\int_{\partial \Omega} \frac{\partial \Phi(x, y)}{\partial \mathbf{n}(y)} v(y) d S(y)=\int_{\partial \Omega} \Phi(x, y) g(y) d S(y) \text { for } x \in \partial \Omega .
$$

The operator on the left of (9) has a one-dimensional kernel, consisting of the constant functions, so we use the fact that $\int_{\partial \Omega} v d S=0$ to obtain a well-posed problem,

$$
\frac{v(x)}{2}+\int_{\partial \Omega}\left(\frac{\partial \Phi(x, y)}{\partial \mathbf{n}(y)}+1\right) v(y) d S(y)=\int_{\partial \Omega} \Phi(x, y) g(y) d S(y) \text { for } x \in \partial \Omega .
$$

As proposed, we solve (10) to obtain $v$ on $\partial \Omega$. Note that

$$
\Phi(x, y)=-(4 \pi)^{-1} \ln |x-y|^{2} \quad, \quad \frac{\partial \Phi(x, y)}{\partial \mathbf{n}(y)}=\frac{(x-y) \cdot \mathbf{n}(y)}{2 \pi|x-y|^{2}}=\frac{(x-y) \cdot(R \mathbf{t}(y))}{2 \pi|x-y|^{2}} .
$$

We parametrize (10) as follows. Let $x=x(t):[0,2 \pi] \rightarrow \partial \Omega$ be a smooth (at least $C^{2}$ ) and $2 \pi$-periodic parametrization of $\partial \Omega$ that traverses the boundary counter-clockwise, such that $\left|x^{\prime}(t)\right| \geq \sigma$ for some $\sigma>0$. Take $V(t)=v(x(t)), G(t)=g(x(t))\left|x^{\prime}(t)\right|$. Since $g=-\partial f / \partial \mathbf{t}$, it holds that $G(t)=-F^{\prime}(t)$, where $F(t)=$ $f(x(t))$. The parametrized form of (10) is

$$
\frac{V(t)}{2}+\int_{0}^{2 \pi} K(t, s) V(s) d s=\int_{0}^{2 \pi} L_{1}(t, s) G(s) d s+\int_{0}^{2 \pi} L_{2}(t, s) G(s) d s \text { for } t \in[0,2 \pi),
$$


where the integral kernels are given by

$$
\begin{aligned}
K(t, s) & =\frac{(x(t)-x(s)) \cdot\left(R x^{\prime}(s)\right)}{2 \pi|x(t)-x(s)|^{2}}+\left|x^{\prime}(s)\right|, \\
L_{1}(t, s) & =-\frac{1}{4 \pi} \ln \left(\frac{|x(t)-x(s)|^{2}}{4 \sin ^{2}((t-s) / 2)}\right), \\
L_{2}(t, s) & =-\frac{1}{4 \pi} \ln \left(4 \sin ^{2}((t-s) / 2)\right) .
\end{aligned}
$$

Simple Taylor series arguments show that the diagonal entries of $K$ and $L_{1}$ are

$$
K(t, t)=\frac{x^{\prime \prime}(t) \cdot\left(R x^{\prime}(t)\right)}{4 \pi\left|x^{\prime}(t)\right|^{2}}+\left|x^{\prime}(t)\right| \quad, \quad L_{1}(t, t)=-\frac{1}{4 \pi} \ln \left|x^{\prime}(t)\right|^{2},
$$

so it is not difficult to see that, for any fixed $t$, both $K(t, s)$ and $L_{1}(t, s)$ are smooth (at least $C^{2}$ ) and $2 \pi$-periodic. It is clear that $L_{2}(t, s)$ is singular, but its singularity is integrable and of a very particular type that is independent of the parametrization.

Nyström discretizations (cf. $[8,11,12]$ ) of (12) involve replacing the integrals with suitable quadratures, and then sampling the corresponding equations at the quadrature points to obtain a square system. The integrals involving $K$ and $L_{1}$ will be treated using the uniform trapezoid rule, which is of high order for (very) smooth periodic functions. Fixing $n$, and taking $t_{j}=\pi j / n$ for $0 \leq j \leq 2 n$,

$$
\int_{0}^{2 \pi} K(t, s) V(s) d s \approx \frac{\pi}{n} \sum_{j=0}^{2 n-1} K\left(t, t_{j}\right) V\left(t_{j}\right) \quad, \quad \int_{0}^{2 \pi} L_{1}(t, s) G(s) d s \approx \frac{\pi}{n} \sum_{j=0}^{2 n-1} L_{1}\left(t, t_{j}\right) G\left(t_{j}\right) .
$$

Following Martensen [10] and Kussmaul [9] (see also [8]), we use a quadrature for the integral involving $L_{2}$ that exploits the specific nature of the singularity,

$$
\int_{0}^{2 \pi} L_{2}(t, s) G(s) d s \approx \sum_{j=0}^{2 n-1} \omega_{j}(t) G\left(t_{j}\right) \quad, \quad \omega_{j}(t)=\frac{1}{2 n} \sum_{m=1}^{n} \frac{\cos \left(m\left(t-t_{j}\right)\right)}{m} .
$$

This quadrature can be derived, for example, by integrating the Fourier series of $G(s)$ term-by-term against $-(4 \pi)^{-1} \ln \left(4 \sin ^{2}((t-s) / 2)\right)$, truncating the resulting series at term $n$, and using the trapezoid rule on $2 n$ subintervals to approximate each of the Fourier coefficients in the finite sum. Key to this derivation is the fact that (cf. [4]),

$$
-\frac{1}{2 \pi} \int_{0}^{2 \pi} \ln \left(4 \sin ^{2}((t-s) / 2)\right) e^{\mathrm{i} m s} d s=\left\{\begin{array}{ll}
0 & , m=0 \\
e^{\mathrm{i} m t} /|m| & , m \neq 0
\end{array} .\right.
$$

We also note that

$$
\omega_{j}\left(t_{i}\right)=\hat{\omega}_{|i-j|} \quad, \quad \hat{\omega}_{k}=\frac{1}{2 n} \sum_{m=1}^{n} \frac{\cos \left(m t_{k}\right)}{m},
$$

which reduces computational costs in computing the weights when integrals involving $L_{2}$ are to be approximated at each $t_{i}$.

The Nyström method corresponding to these quadratures defines the approximations $V_{i} \approx V\left(t_{i}\right)$ as the solution of the linear system

$$
\frac{V_{i}}{2}+\frac{\pi}{n} \sum_{j=0}^{2 n-1} K\left(t_{i}, t_{j}\right) V_{j}=\sum_{j=0}^{2 n-1}\left(\frac{\pi}{n} L_{1}\left(t_{i}, t_{j}\right)+\hat{\omega}_{|i-j|}\right) G\left(t_{j}\right), 0 \leq i \leq 2 n-1 .
$$

Because the $\left\{\left(t_{i}, V_{i}\right)\right\}$ data approximates a smooth periodic function at equispaced points, it is natural to interpolate it with a trigonometric polynomial,

$$
\tilde{V}(t)=\sum_{j=-n}^{n} d_{j} e^{\mathrm{i} j t},
$$




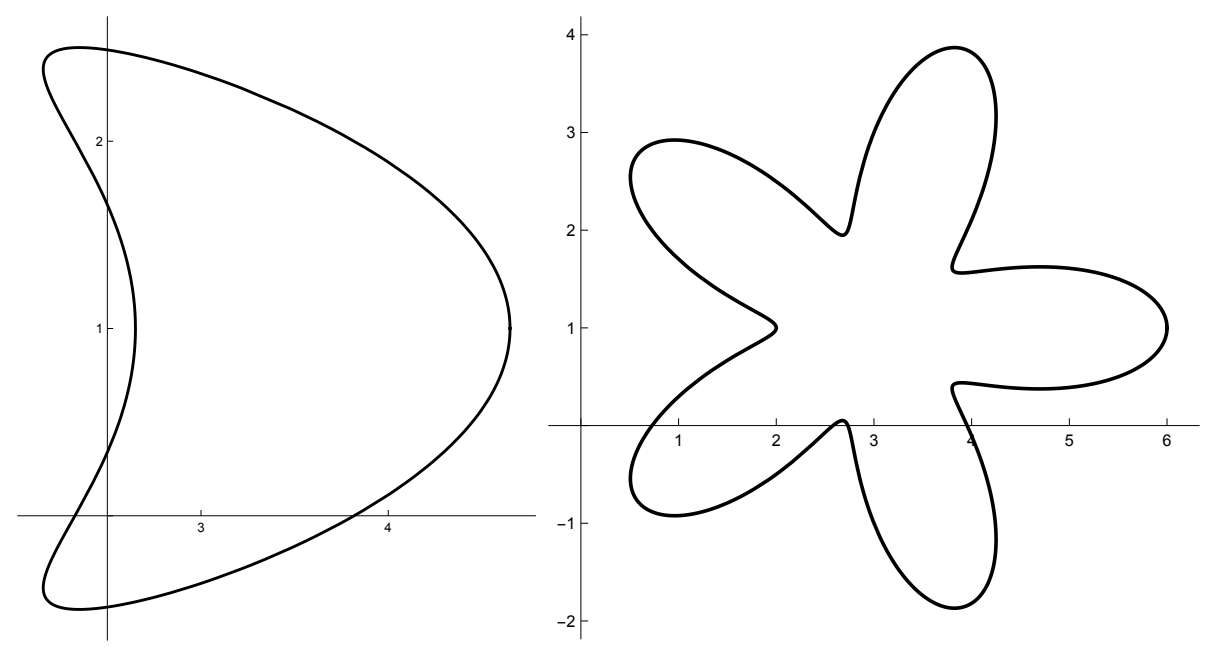

Figure 1. The Bean Domain (left) and the Five-Petal Domain.

for which we use a Fast Fourier Transform (FFT) to efficiently obtain the coefficients $\left\{d_{j}\right\}$. The values $\tilde{V}^{\prime}\left(t_{j}\right)$ are the obtained by an inverse FFT (IFFT), using the coefficients $\left\{\mathfrak{i} j d_{j}\right\}$. From this, we obtain our approximation of the normal derivative of $u$ at $x_{i}=x\left(t_{i}\right)$ as

$$
\frac{\partial u}{\partial \mathbf{n}}\left(x_{i}\right)=\frac{\partial v}{\partial \mathbf{t}}\left(x_{i}\right)=\frac{V^{\prime}\left(t_{i}\right)}{\left|x^{\prime}\left(t_{i}\right)\right|} \approx \frac{\tilde{V}^{\prime}\left(t_{i}\right)}{\left|x^{\prime}\left(t_{i}\right)\right|} .
$$

For $x_{0} \in \Omega$ and its complex counterpart $z_{0}=x_{0} \cdot(1, i)$, the parametrized form of the Cauchy integral formulas (3), and their Nyström-based approximations, are

$$
w^{(k)}\left(z_{0}\right)=\frac{k !}{2 \pi \mathfrak{i}} \int_{0}^{2 \pi} \frac{[F(t)+\mathfrak{i} V(t)] x^{\prime}(t) \cdot(1, \mathfrak{i})}{\left[\left(x(t)-x_{0}\right) \cdot(1, \mathfrak{i})\right]^{k+1}} d t \approx \frac{k !}{2 \pi \mathfrak{i}} \int_{0}^{2 \pi} \frac{[F(t)+\mathfrak{i} \tilde{V}(t)] x^{\prime}(t) \cdot(1, \mathfrak{i})}{\left[\left(x(t)-x_{0}\right) \cdot(1, \mathfrak{i})\right]^{k+1}} d t .
$$

The integral involving $\tilde{V}(t)$ in $(22)$ is also approximated by the trapezoid rule. We already have the data $\left\{V_{j}\right\}$ on hand for the trapezoid rule on $2 n$ subintervals, but we can cheaply double or quadruple (etc.) the number of subintervals via the FFT/IFFT approach to "fill in" data at the new nodes. In several of the experiments in this section and the next, we report how these richer versions of the trapezoid rule compare with the baseline trapezoid rule.

For both examples in this section we use the harmonic conjugate pair

$$
u(x)=\ln |x| \quad, \quad v(x)=\arctan \left(x_{2} / x_{1}\right)-v_{\text {ave }},
$$

where $v_{\text {ave }}$ is the constant such that $\int_{\partial \Omega} v d S=0$. Both domains, which are pictured in Figure 1 , are in right half-plane. These experiments were done in MATLAB, using its built-in "backslash" direct solver for the linear system (19) and FFT/IFFT for computing the trigonmetric interpolant $\tilde{V}$ and its derivative.

Example 2.1 (Bean Shape). We consider the domain $\Omega$ bounded by the curve

$$
x(t)=(\cos t+0.65 \cos (2 t), 1.5 \sin t)+P \quad, \quad P=(3,1) .
$$

In this case, $v_{\text {ave }} \approx 0.286124639031644347606124552$, correct in all digits shown, up to rounding in the last digit. In Table 1 we report various errors with respect the problem-size parameter $n$ from (19). These errors are:

(a) The maximum absolute error in the approximation of $\partial u / \partial \mathbf{n}$ at the nodes $x_{i}=x\left(t_{i}\right)$.

(b) The absolute error in the approximation of $u$, its first partial derivatives and its second partial derivatives at two points in $\Omega$.

For the errors in (b), we experiment with three different levels of resolution for the trapezoid rule in the approximation (22). The lowest level of resolution, indicated by the label $\zeta=1$, uses exactly the data $\left\{\left(t_{i}, V_{i}\right)\right\}$ computed in (19). The next level of resolution, indicated by $\zeta=2$, corresponds to augmenting the 
TABLE 1. Bean: Errors in approximating the normal derivative of $u$ on $\partial \Omega$, and its values and first and second partial derivatives at two points, $P_{1}=(4,1 / 2)$ and $P_{2}=(3,9 / 4)$, in $\Omega$. Below this are the exact values of $u$ and its first and second partial derivatives at these points.

\begin{tabular}{|c|c|c|c|c|}
\hline & & $n=16$ & $n=32$ & $n=64$ \\
\hline & $\partial u / \partial \mathbf{n}$ & $1.4989 \mathrm{e}-04$ & $1.0888 \mathrm{e}-08$ & $9.3328 \mathrm{e}-13$ \\
\hline \multirow{10}{*}{$\zeta=1$} & $u\left(P_{1}\right)$ & $1.8243 \mathrm{e}-03$ & $3.9705 \mathrm{e}-05$ & $5.1622 \mathrm{e}-10$ \\
\hline & $u\left(P_{2}\right)$ & $7.8176 \mathrm{e}-05$ & $1.1441 \mathrm{e}-07$ & $9.3259 \mathrm{e}-15$ \\
\hline & $u_{x_{1}}\left(P_{1}\right)$ & $1.3305 \mathrm{e}-01$ & $1.3028 \mathrm{e}-05$ & $6.8491 \mathrm{e}-08$ \\
\hline & $u_{x_{2}}\left(P_{1}\right)$ & $4.4526 \mathrm{e}-02$ & $1.6231 \mathrm{e}-03$ & $8.3801 \mathrm{e}-08$ \\
\hline & $u_{x_{1}}\left(P_{2}\right)$ & $4.4379 \mathrm{e}-03$ & $1.0111 \mathrm{e}-06$ & $1.2859 \mathrm{e}-13$ \\
\hline & $u_{x_{2}}\left(P_{2}\right)$ & $3.4742 \mathrm{e}-03$ & $3.7146 \mathrm{e}-06$ & $6.9197 \mathrm{e}-13$ \\
\hline & $u_{x_{1} x_{1}}\left(P_{1}\right)$ & $1.8676 \mathrm{e}+00$ & $4.7321 \mathrm{e}-02$ & $7.3981 \mathrm{e}-06$ \\
\hline & $u_{x_{1} x_{2}}\left(P_{1}\right)$ & $1.4898 \mathrm{e}+00$ & $2.9545 \mathrm{e}-02$ & $9.5720 \mathrm{e}-07$ \\
\hline & $u_{x_{1} x_{1}}\left(P_{2}\right)$ & $8.6408 \mathrm{e}-02$ & $8.5098 \mathrm{e}-05$ & $4.1156 \mathrm{e}-11$ \\
\hline & $u_{x_{1} x_{2}}\left(P_{2}\right)$ & 7.1994e-02 & $7.2353 \mathrm{e}-05$ & $1.2868 \mathrm{e}-11$ \\
\hline \multirow{10}{*}{$\zeta=2$} & $u\left(P_{1}\right)$ & $3.9653 \mathrm{e}-05$ & $5.0881 \mathrm{e}-10$ & $4.4409 \mathrm{e}-16$ \\
\hline & $u\left(P_{2}\right)$ & $1.8213 \mathrm{e}-06$ & $1.6698 \mathrm{e}-11$ & $8.8818 \mathrm{e}-16$ \\
\hline & $u_{x_{1}}\left(P_{1}\right)$ & $1.4315 \mathrm{e}-05$ & $6.8485 \mathrm{e}-08$ & $1.0825 \mathrm{e}-15$ \\
\hline & $u_{x_{2}}\left(P_{1}\right)$ & $1.6223 \mathrm{e}-03$ & $8.3802 \mathrm{e}-08$ & $1.7486 \mathrm{e}-15$ \\
\hline & $u_{x_{1}}\left(P_{2}\right)$ & $3.2456 \mathrm{e}-06$ & $1.5828 \mathrm{e}-11$ & $1.6653 \mathrm{e}-16$ \\
\hline & $u_{x_{2}}\left(P_{2}\right)$ & $6.8505 \mathrm{e}-07$ & $4.1912 \mathrm{e}-12$ & 0 \\
\hline & $u_{x_{1} x_{1}}\left(P_{1}\right)$ & $4.7319 \mathrm{e}-02$ & $7.3981 \mathrm{e}-06$ & $3.2960 \mathrm{e}-15$ \\
\hline & $u_{x_{1} x_{2}}\left(P_{1}\right)$ & $2.9546 \mathrm{e}-02$ & $9.5720 \mathrm{e}-07$ & $1.3543 \mathrm{e}-14$ \\
\hline & $u_{x_{1} x_{1}}\left(P_{2}\right)$ & $7.9112 \mathrm{e}-05$ & $4.9585 \mathrm{e}-11$ & $5.3429 \mathrm{e}-16$ \\
\hline & $u_{x_{1} x_{2}}\left(P_{2}\right)$ & $7.2458 \mathrm{e}-05$ & $4.5001 \mathrm{e}-11$ & $1.3878 \mathrm{e}-17$ \\
\hline \multirow{10}{*}{$\zeta=4$} & $u\left(P_{1}\right)$ & $5.2294 \mathrm{e}-08$ & $7.4061 \mathrm{e}-12$ & $1.7764 \mathrm{e}-15$ \\
\hline & $u\left(P_{2}\right)$ & $1.7069 \mathrm{e}-06$ & $1.6707 \mathrm{e}-11$ & $8.8818 \mathrm{e}-16$ \\
\hline & $u_{x_{1}}\left(P_{1}\right)$ & $1.2210 \mathrm{e}-06$ & $5.8367 \mathrm{e}-12$ & $1.9429 \mathrm{e}-16$ \\
\hline & $u_{x_{2}}\left(P_{1}\right)$ & $9.0129 \mathrm{e}-07$ & $1.3441 \mathrm{e}-12$ & $1.4155 \mathrm{e}-15$ \\
\hline & $u_{x_{1}}\left(P_{2}\right)$ & $4.2567 \mathrm{e}-06$ & $1.5956 \mathrm{e}-11$ & $2.4980 \mathrm{e}-16$ \\
\hline & $u_{x_{2}}\left(P_{2}\right)$ & 3.0296e-06 & $4.8828 \mathrm{e}-12$ & $1.3878 \mathrm{e}-16$ \\
\hline & $u_{x_{1} x_{1}}\left(P_{1}\right)$ & $9.0848 \mathrm{e}-06$ & $3.9098 \mathrm{e}-12$ & $1.4433 \mathrm{e}-15$ \\
\hline & $u_{x_{1} x_{2}}\left(P_{1}\right)$ & $1.7443 \mathrm{e}-06$ & $4.1441 \mathrm{e}-13$ & $3.3567 \mathrm{e}-15$ \\
\hline & $u_{x_{1} x_{1}}\left(P_{2}\right)$ & $5.9859 \mathrm{e}-06$ & $8.4289 \mathrm{e}-12$ & $1.0408 \mathrm{e}-16$ \\
\hline & $u_{x_{1} x_{2}}\left(P_{2}\right)$ & $1.0520 \mathrm{e}-07$ & $3.2132 \mathrm{e}-11$ & $4.0246 \mathrm{e}-16$ \\
\hline
\end{tabular}

\begin{tabular}{|c|ccccc|}
\hline & $u\left(P_{j}\right)$ & $u_{x_{1}}\left(P_{j}\right)$ & $u_{x_{2}}\left(P_{j}\right)$ & $u_{x_{1} x_{1}}\left(P_{j}\right)$ & $u_{x_{1} x_{2}}\left(P_{j}\right)$ \\
\hline$P_{1}$ & $1.3940 \mathrm{e}+00$ & $2.4615 \mathrm{e}-01$ & $3.0769 \mathrm{e}-02$ & $-5.9645 \mathrm{e}-02$ & $-1.5148 \mathrm{e}-02$ \\
$P_{2}$ & $1.2524 \mathrm{e}+00$ & $2.4510 \mathrm{e}-01$ & $1.4706 \mathrm{e}-01$ & $-3.8447 \mathrm{e}-02$ & $-7.2088 \mathrm{e}-02$ \\
\hline
\end{tabular}

original data with interpolated data at the midpoints, $t_{i+1 / 2}=\left(t_{i}+t_{i+1}\right) / 2$. The finest level of resolution we will consider, indicated by $\zeta=4$, corresponds to further augmenting the level-two data with interpolated data at the quarter-points, $t_{i+1 / 4}=\left(3 t_{i}+t_{i+1}\right) / 4$ and $t_{i+3 / 4}=\left(t_{i}+3 t_{i+1}\right) / 4$. The computed spectral condition numbers of the matrices associated with (19) remain fixed at $5.9686 \mathrm{e}+00$ for $n=16,32,64$.

The high-order nature of the algorithm is apparent, with significant decreases in each measure of error as the number of points is doubled, reaching machine precision in many cases for $n=64$. We also note that, for this problem, significant reductions in error are achieved for interior values by interpolating the computed data for the trapezoid approximation of (22). 


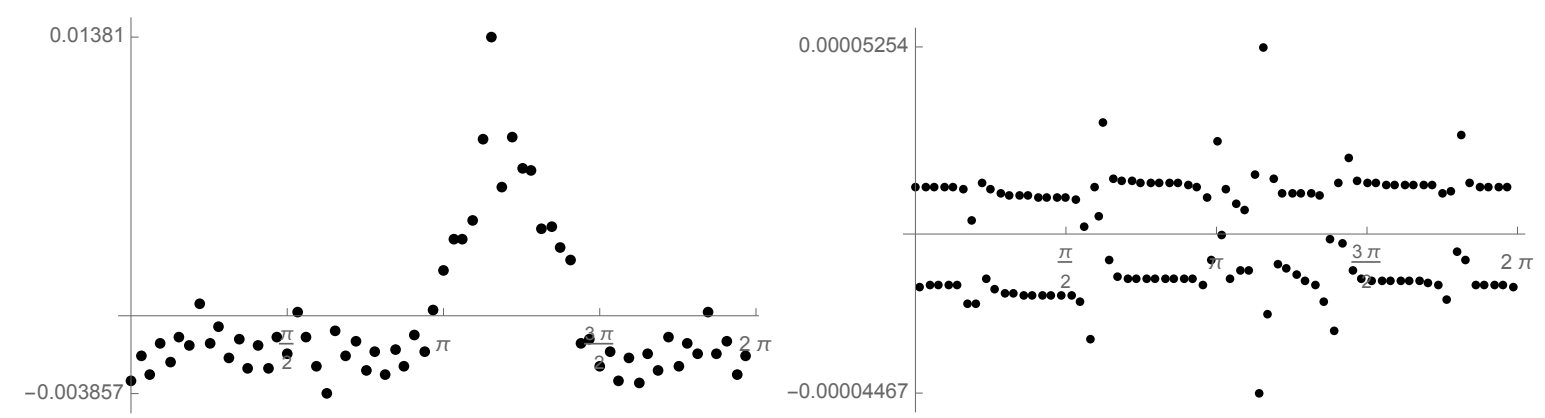

Figure 2. Five-Petal: The errors, $V\left(t_{i}\right)-\tilde{V}\left(t_{i}\right)$, in approximating the Dirichlet trace of $v$ for $n=32$ (left) and $n=64$.

TABLE 2. Five-Petal: Errors in approximating the normal derivative of $u$ on $\partial \Omega$, and its values and first and second partial derivatives at two points, $P_{1}=(2,1 / 2)$ and $P_{2}=(7 / 2,1)$, in $\Omega$. Below this are the exact values of $u$ and its first and second partial derivatives at these points.

\begin{tabular}{|c|c|ccccc|}
\hline & & $n=16$ & $n=32$ & $n=64$ & $n=128$ & $n=256$ \\
\hline & $\partial u / \partial \mathbf{n}$ & $5.5351 \mathrm{e}-01$ & $1.2496 \mathrm{e}-01$ & $3.5390 \mathrm{e}-03$ & $3.4062 \mathrm{e}-6$ & $2.9735 \mathrm{e}-12$ \\
\hline \multirow{5}{*}{$\zeta=2$} & $u\left(P_{1}\right)$ & $2.1926 \mathrm{e}-02$ & $8.1485 \mathrm{e}-04$ & $6.8438 \mathrm{e}-07$ & $1.0090 \mathrm{e}-11$ & $5.5511 \mathrm{e}-16$ \\
& $u\left(P_{2}\right)$ & $2.1976 \mathrm{e}-03$ & $4.2675 \mathrm{e}-05$ & $2.1450 \mathrm{e}-06$ & $2.9139 \mathrm{e}-11$ & $1.5543 \mathrm{e}-15$ \\
& $u_{x_{1}}\left(P_{1}\right)$ & $4.6939 \mathrm{e}-02$ & $2.4008 \mathrm{e}-03$ & $2.1684 \mathrm{e}-06$ & $5.0886 \mathrm{e}-11$ & $2.7756 \mathrm{e}-16$ \\
& $u_{x_{2}}\left(P_{1}\right)$ & $3.3170 \mathrm{e}-02$ & $2.2415 \mathrm{e}-03$ & $2.1590 \mathrm{e}-07$ & $5.0079 \mathrm{e}-11$ & $2.0817 \mathrm{e}-16$ \\
& $u_{x_{1}}\left(P_{2}\right)$ & $9.0903 \mathrm{e}-03$ & $2.9921 \mathrm{e}-06$ & $1.9011 \mathrm{e}-06$ & $5.9653 \mathrm{e}-11$ & $2.7756 \mathrm{e}-16$ \\
& $u_{x_{2}}\left(P_{2}\right)$ & $1.6139 \mathrm{e}-02$ & $6.4784 \mathrm{e}-04$ & $1.0570 \mathrm{e}-08$ & $2.8182 \mathrm{e}-11$ & $3.8858 \mathrm{e}-16$ \\
& $u_{x_{1} x_{1}}\left(P_{1}\right)$ & $1.1008 \mathrm{e}-01$ & $4.4535 \mathrm{e}-03$ & $3.8472 \mathrm{e}-06$ & $2.1866 \mathrm{e}-10$ & $6.3838 \mathrm{e}-16$ \\
& $u_{x_{1} x_{2}}\left(P_{1}\right)$ & $1.0526 \mathrm{e}-01$ & $1.3734 \mathrm{e}-04$ & $6.2953 \mathrm{e}-06$ & $2.0828 \mathrm{e}-11$ & $1.4710 \mathrm{e}-15$ \\
& $u_{x_{1} x_{1}}\left(P_{2}\right)$ & $1.3452 \mathrm{e}-02$ & $2.9259 \mathrm{e}-04$ & $3.5473 \mathrm{e}-06$ & $2.0290 \mathrm{e}-10$ & $8.3267 \mathrm{e}-17$ \\
& $u_{x_{1} x_{2}}\left(P_{2}\right)$ & $1.2613 \mathrm{e}-02$ & $4.0277 \mathrm{e}-04$ & $6.4810 \mathrm{e}-07$ & $1.8877 \mathrm{e}-11$ & $2.4286 \mathrm{e}-16$ \\
\hline
\end{tabular}

\begin{tabular}{|c|ccccc|}
\hline & $u\left(P_{j}\right)$ & $u_{x_{1}}\left(P_{j}\right)$ & $u_{x_{2}}\left(P_{j}\right)$ & $u_{x_{1} x_{1}}\left(P_{j}\right)$ & $u_{x_{1} x_{2}}\left(P_{j}\right)$ \\
\hline$P_{1}$ & $7.2346 \mathrm{e}-01$ & $4.7059 \mathrm{e}-01$ & $1.1765 \mathrm{e}-01$ & $-2.0761 \mathrm{e}-01$ & $-1.1073 \mathrm{e}-01$ \\
$P_{2}$ & $1.2920 \mathrm{e}+00$ & $2.6415 \mathrm{e}-01$ & $7.5472 \mathrm{e}-02$ & $-6.4080 \mathrm{e}-02$ & $-3.9872 \mathrm{e}-02$ \\
\hline
\end{tabular}

Example 2.2 (Five-Petal Shape). We consider the domain $\Omega$ bounded by the curve

$$
x(t)=(2+\cos (m t))(\cos t, \sin t)+P \quad, \quad m=5 \quad, \quad P=(3,1) .
$$

We use the same harmonic conjugates as were used in the Bean example, Example 2.1. In this case, $v_{\text {ave }} \approx 0.289910246091888587979329869$, correct in all digits shown. Plots of the errors, $V\left(t_{i}\right)-\tilde{V}\left(t_{i}\right)$, in the approximations of the Dirichlet trace of $v$, computed from the Nyström linear system (19) when $n=32$ and $n=64$, are given in Figure 2. For $n=128$, the magnitude of maximal error is approximately $1.083 \times 10^{-8}$, while the mean magnitude of the errors is approximately $3.222 \times 10^{-9}$. We report the same kinds of errors as in the Bean example, for the choice $\zeta=2$, in Table 2. There was modest improvement in errors by increasing from $\zeta=1$ to $\zeta=2$, but no further improvement for $\zeta=4$. Because of the greater curvature of the domain, more points are needed to reach machine precision, but the same high-order convergence is clear. The computed spectral condition numbers of the associated matrices monotonically decrease from $9.7013 \mathrm{e}+00$ when $n=16$ to $9.0344 \mathrm{e}+00$ when $n=256$. 


\section{Piecewise Smooth Boundary and Data}

We briefly describe the key difficulties that arise when $\Omega$ has corners. The first is that neither of the integrands in (10) is a smooth periodic function for fixed $x$. More specifically, if $z \in \partial \Omega$ is a corner point where the interior angle is $\pi / \alpha$, and $x, y \in \partial \Omega$ are on opposite sides of the corner, then

$$
\lim _{y \rightarrow z} \frac{\partial \Phi(x, y)}{\partial \mathbf{n}(y)}=\frac{\sin (\pi / \alpha)}{2 \pi|x-z|} .
$$

This is clearly unbounded as $x \rightarrow z$. Additionally, $u$ and $v$ are typically only Hölder continous near $z$, with unbounded partial derivatives (cf. $[3,20,21])$. Again, more specifically,

$$
|u(x)-u(z)| \sim a|x-z|^{\alpha} \text { for } x \in \bar{\Omega} \quad, \quad|\partial u / \partial \mathbf{n}(x)| \sim b|x-z|^{\alpha-1} \text { for } x \in \partial \Omega,
$$

for some constants $a, b$ that are typically non-zero. The same holds for $v$. If the corner angle at $z$ is obtuse, $0<\alpha<1$, then we expect the normal derivatives of $u$ and $v$ to be unbounded near $z$. Because of the assumption that $u$ has piecewise smooth Dirichlet data, its tangential derivative will be bounded near $z$, but the tangential derivative of $v$, which is the normal derivative of $u$, will typically be unbounded in the case of obtuse angles. This singular behavior of $u, v$ is illustrated in the Teardrop and Boomerang examples below (cf. $[1,7]$ ), both of which have a single corner, at the origin, with angle $\pi / \alpha$, see Figure 3 . For these examples we take the harmonic conjugate pair

$$
u=r^{\alpha} \cos (\alpha \theta) \quad, \quad v=r^{\alpha} \sin (\alpha \theta),
$$

where $r=|x|$ and $\theta$ are the standard polar coordinates.

The second second difficulty is that the integral equation (10) is no longer valid, when viewed pointwise, at corners. It is well-known that

$$
\int_{\partial \Omega} \frac{\partial \Phi(x, y)}{\partial \mathbf{n}(y)} d s(y)=\left\{\begin{array}{ll}
-1 / 2 & , x \in \partial \Omega \text { not at corner } \\
-1 /(2 \alpha) & , x \in \partial \Omega \text { at } \pi / \alpha \text { corner }
\end{array},\right.
$$

and a consequence of this is that, for $z \in \partial \Omega$ a corner with interior angle $\pi / \alpha$,

$$
\frac{v(z)}{2 \alpha}+\int_{\partial \Omega}\left(\frac{\partial \Phi(z, y)}{\partial \mathbf{n}(y)}+1\right) v(y) d S(y)=\int_{\partial \Omega} \Phi(z, y) g(y) d S(y) .
$$

A simple modification removes the $\alpha$-dependency in the integral operator (cf. [7]),

$$
|\partial \Omega| v(z)+\frac{v(x)-v(z)}{2}+\int_{\partial \Omega}\left(\frac{\partial \Phi(x, y)}{\partial \mathbf{n}(y)}+1\right)(v(y)-v(z)) d S(y)=\int_{\partial \Omega} \Phi(x, y) g(y) d S(y),
$$

for $x=z$ and any $x \in \partial \Omega$ that is not at a corner. If $z$ is the only corner, we may take (26) for all $x \in \partial \Omega$. We will focus on that case for much of the discussion of how the approach from Section 2 must be modified. After developing an approach to treat domains with a single corner, we will briefly describe how it can be adjusted to accommodate multiple corners.

3.1. Domains with a Single Corner. Taking a smooth parametrization $x=x(t):[0,2 \pi] \rightarrow \partial \Omega$ of $\partial \Omega$ as before, with $x(0)=x(2 \pi)=z$, and using the same notation as in Section 2, the parametrized version of (26) is

$$
|\partial \Omega| V(0)+\frac{V(t)-V(0)}{2}+\int_{0}^{2 \pi} K(t, s)(V(s)-V(0)) d s=\int_{0}^{2 \pi}\left(L_{1}(t, s)+L_{2}(t, s)\right) G(s) d s
$$

Both integrands in (27) are more challenging than in the smooth case, but both will be addressed by essentially the same quadratures as before (trapezoid and Martensen), after a suitable change-of-variable.

The relevant quadrature for the first integral in (27) is analyzed in [7], and we briefly describe it for a generic interval $[a, b]$. Given an integer $p \geq 2$, let $\lambda:[a, b] \rightarrow[a, b]$ be given by

$$
\lambda(t)=(b-a) \frac{[c(t)]^{p}}{[c(t)]^{p}+[1-c(t)]^{p}}+a \quad, \quad c(t)=\left(\frac{1}{2}-\frac{1}{p}\right)\left(\frac{2 t-a-b}{b-a}\right)^{3}+\frac{1}{p}\left(\frac{2 t-a-b}{b-a}\right)+\frac{1}{2} .
$$



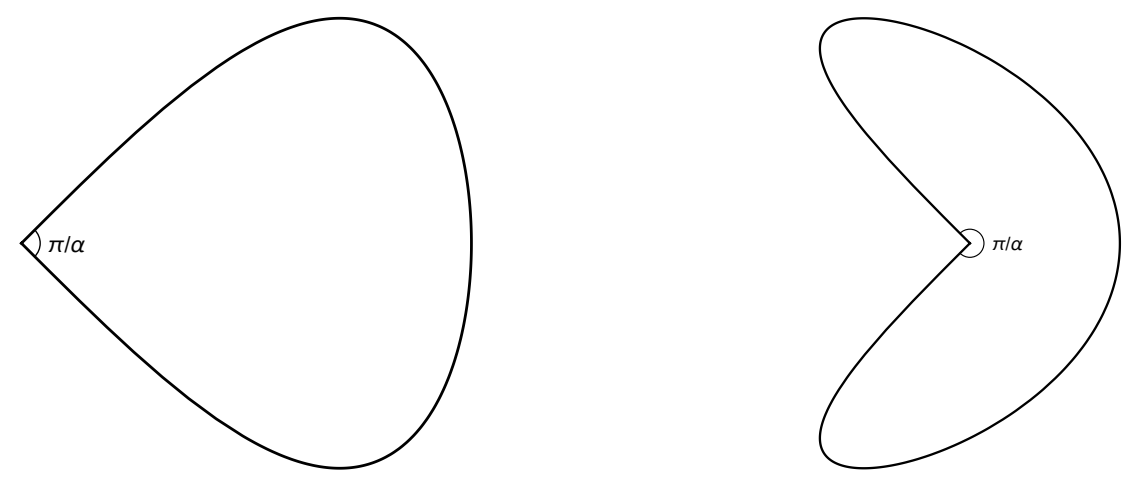

$u$ - --n- $v$

$u---\boldsymbol{- n} v$
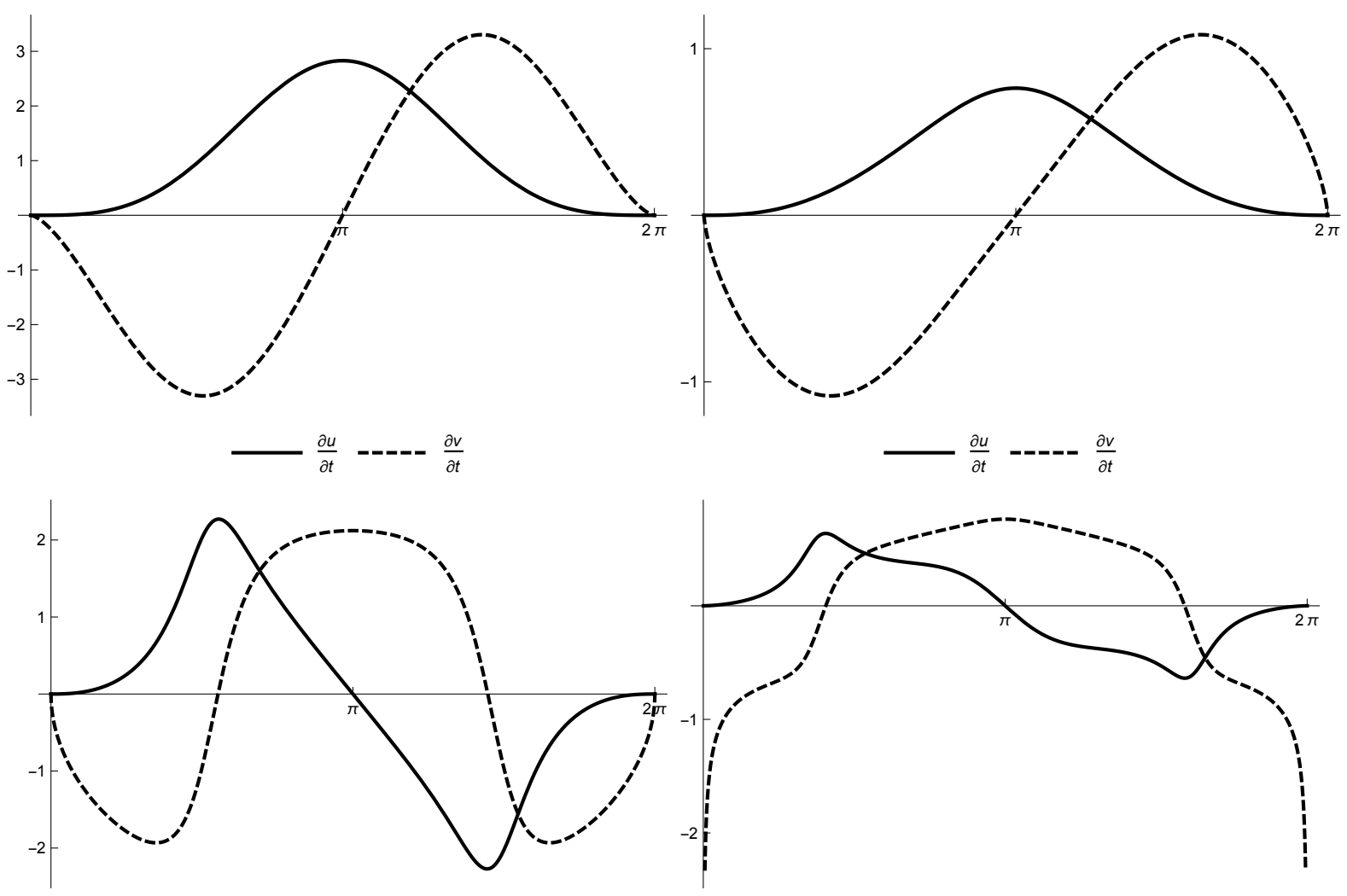

Figure 3. The Teardrop (left) and Boomerang domains. Below each domain are parametric plots of $u, v$ and $\partial u / \partial \mathbf{t}, \partial v / \partial \mathbf{t}$ for the conjugate pair from (24). For the Teardrop, $\alpha=3 / 2$. For the Boomerang, $\alpha=2 / 3$.

The function $\lambda$ is strictly increasing, and $\lambda(t)-a$ and $b-\lambda(t)$ have roots of multiplicity $p$ at $a$ and $b$, respectively. Letting $h=(b-a) / m$ and $\tau_{k}=a+k h$, an $(m+1)$-point quadrature associated with this is based on the change of variable $t=\lambda(\tau)$,

$$
\int_{a}^{b} F(t) d t=\int_{a}^{b} F(\tau) \lambda^{\prime}(\tau) d \tau \approx \sum_{k=0}^{m} w_{k} F\left(t_{k}\right) \quad, \quad t_{k}=\lambda\left(\tau_{k}\right) \quad, \quad w_{k}=h \lambda^{\prime}\left(\tau_{k}\right) .
$$

Here $F \in C(a, b)$ is integrable, and typically very smooth in $(a, b)$. Since $\lambda^{\prime}(a)=\lambda^{\prime}(b)=0$, we can omit the points/weights $\left(t_{0}, w_{0}\right)$ and/or $\left(t_{m}, w_{m}\right)$, and this is necessary when $F$ is unbounded at $a$ or $b$. In very 
TABLE 3. Errors in the quadrature (29) for $\int_{-1}^{1} F(t) d t$ for some singular and "nearly singular" $F$. For the final, "nearly singular", integrand, Gauss-Legendre quadture errors are given for comparison.

\begin{tabular}{|r|cc|cc|ccc|}
\hline & \multicolumn{2}{|c|}{$F(t)=\left(1-t^{2}\right)^{1 / 3}$} & \multicolumn{2}{c|}{$F(t)=\left(1-t^{2}\right)^{-1 / 3}$} & \multicolumn{3}{c|}{$F(t)=\left(1+10^{-4}-t^{2}\right)^{-1 / 2}$} \\
\hline$m$ & $p=5$ & $p=7$ & $p=5$ & $p=7$ & $p=5$ & $p=7$ & Gauss \\
\hline 2 & $3.174 \mathrm{e}-01$ & $3.174 \mathrm{e}-01$ & $5.871 \mathrm{e}-01$ & $5.871 \mathrm{e}-01$ & $1.122 \mathrm{e}+00$ & $1.122 \mathrm{e}+00$ & $6.722 \mathrm{e}-01$ \\
4 & $1.020 \mathrm{e}-01$ & $2.579 \mathrm{e}-01$ & $4.682 \mathrm{e}-02$ & $1.945 \mathrm{e}-02$ & $5.924 \mathrm{e}-03$ & $1.181 \mathrm{e}-01$ & $3.665 \mathrm{e}-01$ \\
8 & $4.213 \mathrm{e}-04$ & $2.057 \mathrm{e}-03$ & $1.557 \mathrm{e}-03$ & $5.915 \mathrm{e}-04$ & $7.467 \mathrm{e}-03$ & $1.242 \mathrm{e}-02$ & $1.854 \mathrm{e}-01$ \\
16 & $5.184 \mathrm{e}-08$ & $1.863 \mathrm{e}-07$ & $1.341 \mathrm{e}-04$ & $5.415 \mathrm{e}-06$ & $3.412 \mathrm{e}-03$ & $1.089 \mathrm{e}-03$ & $8.678 \mathrm{e}-02$ \\
32 & $7.938 \mathrm{e}-10$ & $7.244 \mathrm{e}-12$ & $1.246 \mathrm{e}-05$ & $2.600 \mathrm{e}-07$ & $6.863 \mathrm{e}-06$ & $2.188 \mathrm{e}-05$ & $3.607 \mathrm{e}-02$ \\
64 & $9.114 \mathrm{e}-12$ & $9.104 \mathrm{e}-15$ & $1.197 \mathrm{e}-06$ & $1.112 \mathrm{e}-08$ & $8.817 \mathrm{e}-08$ & $9.773 \mathrm{e}-09$ & $1.181 \mathrm{e}-02$ \\
128 & $9.570 \mathrm{e}-14$ & $4.441 \mathrm{e}-16$ & $1.168 \mathrm{e}-07$ & $4.535 \mathrm{e}-10$ & $3.137 \mathrm{e}-10$ & $4.268 \mathrm{e}-13$ & $2.260 \mathrm{e}-03$ \\
256 & 0 & $8.882 \mathrm{e}-16$ & $1.150 \mathrm{e}-08$ & $2.301 \mathrm{e}-11$ & $4.914 \mathrm{e}-12$ & $1.332 \mathrm{e}-15$ & $1.271 \mathrm{e}-04$ \\
512 & $2.220 \mathrm{e}-16$ & $4.441 \mathrm{e}-16$ & $1.135 \mathrm{e}-09$ & $1.245 \mathrm{e}-10$ & $7.860 \mathrm{e}-14$ & $7.638 \mathrm{e}-14$ & $5.468 \mathrm{e}-07$ \\
1024 & $1.332 \mathrm{e}-15$ & 0 & $1.143 \mathrm{e}-10$ & $6.871 \mathrm{e}-11$ & $1.776 \mathrm{e}-15$ & $3.464 \mathrm{e}-14$ & $1.390 \mathrm{e}-11$ \\
\hline
\end{tabular}

general terms, increasing $p$ increases the order of the quadrature, but for a fixed $m$ a smaller $p$ may give a better result. Because this quadrature may be unfamiliar, we illustrate its performance in Table 3 on a few integrals, $\int_{-1}^{1} F(t) d t$, that are challenging for many quadratures. The first integrand, $F(t)=\left(1-t^{2}\right)^{1 / 3}$, has stronger singular behavior than what would arise in the integration of $u, v$ along $\partial \Omega$. The second integrand, $F(t)=\left(1-t^{2}\right)^{-1 / 3}$, has stronger singular behavior than what would arise in the integration of the tangential or normal derivatives of $u, v$ along $\partial \Omega$. Though one could, in principle, derive Gaussian quadratures integrals involving these kinds of singularities, the approach (29) does not require a priori knowledge of the particular kind of singularity, and is effective on a wide range of integrands by adjusting $m$ and/or $p$, so it is more of a multi-purpose quadrature scheme. The third integrand, $F(t)=\left(1+10^{-4}-t^{2}\right)^{-1 / 2}$, has singularities just outside the interval of integration, and we refer to it as being "nearly singular", because its extremely large derivatives near \pm 1 make it essentially singular for numerical purposes. The integrand $F(s)=K(t, s)(V(s)-V(0))$ in (27), exhibits similar nearly singular behavior for $s$ near $2 \pi$ when $t$ is close to 0 , and for $s$ near 0 when $t$ is close to $2 \pi$. For the final integrand, we compare the performance of (29) with Gauss-Legendre quadrature.

For integrands of the form $F(t)=\left(1-t^{2}\right)^{q} Q(t)$, where $q>-1$ and $Q \in C^{\infty}(-1,1) \cap C[-1,1]$, one can see that the integrand after the change-of-variable $t=\lambda(\tau)$ has the form,

$$
F(\lambda(\tau)) \lambda^{\prime}(\tau)=\left(1-\tau^{2}\right)^{p(q+1)-1} R(\tau)
$$

for some $R \in C^{\infty}(-1,1) \cap C[-1,1]$. It is well-known (cf. [16]) that error in the $(m+1)$-point trapezoid rule decays like the $m^{t h}$ Fourier coefficient of the integrand. In our case, that error is $\mathcal{O}\left(m^{-p(q+1)}\right)$, which is consistent with the convergence behavior seen for the first two integrands in Table 3, until the effects of double-precision arithmetic begin to dominate. This kind of analysis yields a slightly stronger convergence result than that given in [7].

The quadrature (29) will be used to approximate the integral on the left-hand side of (27), so we must approximate the integral on the right-hand side at these quadrature points, $t_{k}=\lambda\left(\tau_{k}\right)$. After the changeof-variable $t=\lambda(\tau), s=\lambda(\sigma)$, the integral on the right-hand side of (27) becomes

$$
\begin{aligned}
J(\tau)= & -\frac{1}{4 \pi} \int_{0}^{2 \pi} \ln \left(|x(\lambda(\tau))-x(\lambda(\sigma))|^{2}\right) G(\lambda(\sigma)) \lambda^{\prime}(\sigma) d \sigma \\
= & -\frac{1}{4 \pi} \int_{0}^{2 \pi} \ln \left(\frac{|x(\lambda(\tau))-x(\lambda(\sigma))|^{2}}{4 \sin ^{2}((\tau-\sigma) / 2)}\right) G(\lambda(\sigma)) \lambda^{\prime}(\sigma) d \sigma \\
& -\frac{1}{4 \pi} \int_{0}^{2 \pi} \ln \left(4 \sin ^{2}((\tau-\sigma) / 2)\right) G(\lambda(\sigma)) \lambda^{\prime}(\sigma) d \sigma,
\end{aligned}
$$




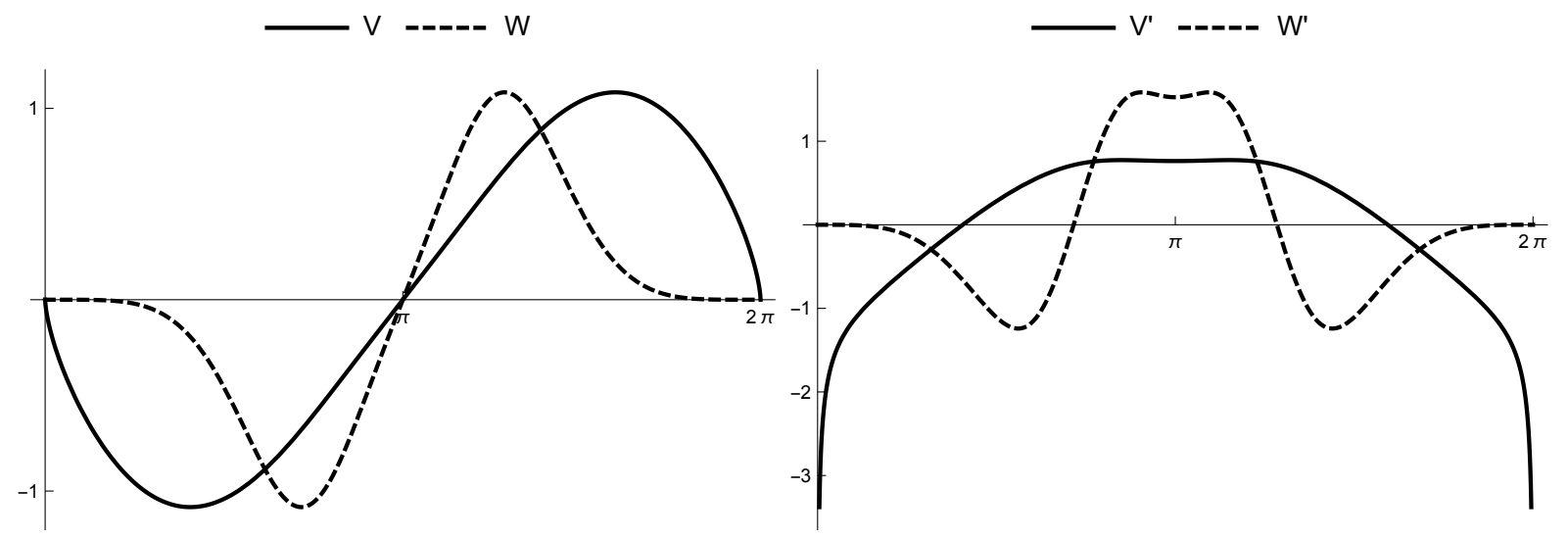

Figure 4. Plots of $V$ and $W$, and $V^{\prime}$ and $W^{\prime}$, for the Boomerang, with $\alpha=2 / 3, p=7$ and $v=r^{\alpha} \sin (\alpha \theta)$.

and we write $J(\tau)=J_{1}(\tau)+J_{2}(\tau)$ to correspond to this decomposition of the integral. The first of these, $J_{1}(t)$, is handled via the trapezoid rule in $\sigma$, which corresponds to $(29)$ in $s$. We note that

$$
\lim _{\sigma \rightarrow \tau} \tilde{L}_{1}(\tau, \sigma)=2 \ln \left(\left|x^{\prime}(\lambda(\tau))\right| \lambda^{\prime}(\tau)\right) \quad \text { where } \quad \tilde{L}_{1}(\tau, \sigma)=\ln \left(\frac{|x(\lambda(\tau))-x(\lambda(\sigma))|^{2}}{4 \sin ^{2}((\tau-\sigma) / 2)}\right)
$$

The integral $J_{2}(\tau)$ is handled by Martensen quadrature, as before. These quadrature choices yield a natural analogue of the Nyström linear system (19), given by

$$
|\partial \Omega| V_{0}+\frac{V_{i}-V_{0}}{2}+\sum_{j=0}^{2 n-1} K\left(t_{i}, t_{j}\right) w_{j}\left(V_{j}-V_{0}\right)=\sum_{j=0}^{2 n-1}\left(\tilde{L}_{1}\left(\tau_{i}, \tau_{j}\right) w_{j}+\hat{\omega}_{|i-j|} \lambda^{\prime}\left(\tau_{j}\right)\right) G\left(t_{j}\right),
$$

for $0 \leq i \leq 2 n-1$, where $w_{j}, t_{j}, \tau_{j}$ are described between $(28)$ and $(29)$, with $(a, b)=(0,2 \pi)$ and $m=2 n-1$. Since $w_{j}=(\pi / n) \lambda^{\prime}\left(\tau_{j}\right)$ in this case, we may re-express the terms in the second sum as

$$
\left(\tilde{L}_{1}\left(\tau_{i}, \tau_{j}\right) w_{j}+\hat{\omega}_{|i-j|} \lambda^{\prime}\left(\tau_{j}\right)\right) G\left(t_{j}\right)=\left(\tilde{L}_{1}\left(\tau_{i}, \tau_{j}\right)+(n / \pi) \hat{\omega}_{|i-j|}\right) w_{j} G\left(t_{j}\right)
$$

The structure of the linear system (31) suggests that an iterative linear solver such as GMRES [15] is a natural choice, and that is what we use in our experiments below.

Let $W(\tau)=V(\lambda(\tau))=V(t)$. The values $V_{i} \approx V\left(t_{i}\right)=W\left(\tau_{i}\right)$ are associated with an even spacing in $\tau$, not $t$, so it makes sense to use the data $\left\{\left(\tau_{i}, V_{i}\right)\right\}$ to construct a trigonometric polynomial $\tilde{W}(\tau) \approx W(\tau)$ and evaluate it and its derivative using the same FFT/IFFT approach as in the smooth boundary case. We have the obvious relationship, for $x=x(t)$,

$$
\frac{\partial u}{\partial \mathbf{n}}(x)=\frac{\partial v}{\partial \mathbf{t}}(x)=\frac{V^{\prime}(t)}{\left|x^{\prime}(t)\right|}=\frac{W^{\prime}(\tau)}{\left|x^{\prime}(t)\right| \lambda^{\prime}(\tau)}
$$

Working with $W$, instead of $V$, is very natural for a few reasons. The first is that $W$ has greater Hölder regularity than $V$. More specifically, $V^{\prime}$ is typically unbounded near 0 and $2 \pi$ when the interior angle at the corner is obtuse, whereas $W^{\prime}$ is not. This is illustrated in Figure 4 for the Boomerang domain (see Figure 3 and Example 3.2), with $\alpha=2 / 3, p=7$ and $v=r^{\alpha} \sin (\alpha \theta)$. Moreover, for sufficiently smooth $\psi$ we have

$$
\int_{\Omega} \nabla u(x) \cdot \nabla \psi(x) d x=\int_{\partial \Omega} \frac{\partial u}{\partial \mathbf{n}}(x) \psi(x) d S(x)=\int_{0}^{2 \pi} V^{\prime}(t) \tilde{\psi}(t) d t=\int_{0}^{2 \pi} W^{\prime}(\tau) \tilde{\psi}(\lambda(\tau)) d \tau
$$

where $\tilde{\psi}(t)=\psi(x(t))$. For example, taking $\psi=u$, we may obtain an accurate approximation of the $H^{1}(\Omega)$ seminorm of $u$ by computing the boundary integral. 
Example 3.1 (Teardrop Domain). The boundary of the Teardrop domain is parametrized by

$$
x(t)=(2 \sin (t / 2),-\beta \sin t) \quad, \quad \beta=\tan (\pi /(2 \alpha)),
$$

for some fixed $\alpha>1$, and $t \in[0,2 \pi)$. Choosing $\alpha=3 / 2$ results in $|\partial \Omega| \approx 8.488331067320425334480158779233$, correct to all digits shown. Since $\partial u / \partial \mathbf{n}$ is unbounded, in Table 4 we instead report the maximal error in the approximation of $W^{\prime}$ at the nodes $x_{i}=x\left(\lambda\left(\tau_{i}\right)\right)$, along with the errors in the approximations of $u$ and those of its derivatives at two test points, for $n=32,64,128$. Because increasing the interpolation parameter $\zeta$ yields some improvements in the approximation of $u$ and its derivatives in the interior, we report the results for $\zeta=1,2,4$. In each case we fix the Kress quadrature parameter $p=7$.

TABLE 4. Teardrop: Errors in approximating $W^{\prime}(\tau)$ on $\partial \Omega$, and the values of $u$ and its first and second partial derivatives at two points, $P_{1}=(1 / 4,-1 / 4)$ and $P_{2}=(3 / 2,1 / 2)$, in $\Omega$. Below this are the exact values of $u$ and its first and second partial derivatives at these points.

\begin{tabular}{|c|c|c|c|c|}
\hline & & $n=32$ & $n=64$ & $n=128$ \\
\hline & $W^{\prime}(\tau)$ & $2.4757 \mathrm{e}-08$ & $1.1834 \mathrm{e}-10$ & $2.2128 \mathrm{e}-11$ \\
\hline \multirow{10}{*}{$\zeta=1$} & $u\left(P_{1}\right)$ & $7.9294 \mathrm{e}-04$ & $3.6510 \mathrm{e}-05$ & $7.5064 \mathrm{e}-09$ \\
\hline & $u\left(P_{2}\right)$ & $9.5304 \mathrm{e}-05$ & $2.8453 \mathrm{e}-09$ & $8.5709 \mathrm{e}-14$ \\
\hline & $u_{x_{1}}\left(P_{1}\right)$ & $1.2030 \mathrm{e}-01$ & $4.1148 \mathrm{e}-03$ & $2.5001 \mathrm{e}-07$ \\
\hline & $u_{x_{1}}\left(P_{2}\right)$ & $2.0874 \mathrm{e}-03$ & $1.2807 \mathrm{e}-07$ & $2.3204 \mathrm{e}-13$ \\
\hline & $u_{x_{2}}\left(P_{1}\right)$ & 6.7333e-02 & $5.1754 \mathrm{e}-04$ & $1.7720 \mathrm{e}-06$ \\
\hline & $u_{x_{2}}\left(P_{2}\right)$ & $2.5234 \mathrm{e}-03$ & $4.8239 \mathrm{e}-07$ & $9.4480 \mathrm{e}-14$ \\
\hline & $u_{x_{1} x_{1}}\left(P_{1}\right)$ & $6.0023 \mathrm{e}+00$ & $2.4731 \mathrm{e}-01$ & $1.8116 \mathrm{e}-04$ \\
\hline & $u_{x_{1} x_{1}}\left(P_{2}\right)$ & $4.5915 \mathrm{e}-02$ & $5.7993 \mathrm{e}-06$ & $1.9174 \mathrm{e}-13$ \\
\hline & $u_{x_{1} x_{2}}\left(P_{1}\right)$ & $1.4401 \mathrm{e}+00$ & $2.8889 \mathrm{e}-01$ & $2.7358 \mathrm{e}-04$ \\
\hline & $u_{x_{1} x_{2}}\left(P_{2}\right)$ & $5.1692 \mathrm{e}-02$ & $1.9819 \mathrm{e}-05$ & $1.3299 \mathrm{e}-13$ \\
\hline \multirow{10}{*}{$\zeta=2$} & $u\left(P_{1}\right)$ & $3.6509 \mathrm{e}-05$ & $7.5065 \mathrm{e}-09$ & $1.7902 \mathrm{e}-13$ \\
\hline & $u\left(P_{2}\right)$ & $2.7874 \mathrm{e}-09$ & $7.8826 \mathrm{e}-14$ & $8.4155 \mathrm{e}-14$ \\
\hline & $u_{x_{1}}\left(P_{1}\right)$ & $4.1148 \mathrm{e}-03$ & $2.5002 \mathrm{e}-07$ & $4.8361 \mathrm{e}-13$ \\
\hline & $u_{x_{1}}\left(P_{2}\right)$ & $1.2798 \mathrm{e}-07$ & $2.1649 \mathrm{e}-13$ & $2.3292 \mathrm{e}-13$ \\
\hline & $u_{x_{2}}\left(P_{1}\right)$ & $5.1754 \mathrm{e}-04$ & $1.7720 \mathrm{e}-06$ & $7.9381 \mathrm{e}-14$ \\
\hline & $u_{x_{2}}\left(P_{2}\right)$ & $4.8240 \mathrm{e}-07$ & $2.2787 \mathrm{e}-13$ & $9.8588 \mathrm{e}-14$ \\
\hline & $u_{x_{1} x_{1}}\left(P_{1}\right)$ & $2.4731 \mathrm{e}-01$ & $1.8116 \mathrm{e}-04$ & $8.1177 \mathrm{e}-12$ \\
\hline & $u_{x_{1} x_{1}}\left(P_{2}\right)$ & 5.7994e-06 & $1.7342 \mathrm{e}-13$ & $7.6605 \mathrm{e}-14$ \\
\hline & $u_{x_{1} x_{2}}\left(P_{1}\right)$ & $2.8889 \mathrm{e}-01$ & $2.7358 \mathrm{e}-04$ & $5.8813 \mathrm{e}-11$ \\
\hline & $u_{x_{1} x_{2}}\left(P_{2}\right)$ & $1.9819 \mathrm{e}-05$ & $6.1282 \mathrm{e}-13$ & $5.4222 \mathrm{e}-13$ \\
\hline \multirow{10}{*}{$\zeta=4$} & $u\left(P_{1}\right)$ & $7.4665 \mathrm{e}-09$ & $2.8486 \mathrm{e}-13$ & $1.7918 \mathrm{e}-13$ \\
\hline & $u\left(P_{2}\right)$ & $5.7809 \mathrm{e}-11$ & $7.8604 \mathrm{e}-14$ & $8.2157 \mathrm{e}-14$ \\
\hline & $u_{x_{1}}\left(P_{1}\right)$ & $2.4997 \mathrm{e}-07$ & $1.1471 \mathrm{e}-12$ & $5.8287 \mathrm{e}-13$ \\
\hline & $u_{x_{1}}\left(P_{2}\right)$ & $9.7181 \mathrm{e}-11$ & $2.2204 \mathrm{e}-13$ & $2.3470 \mathrm{e}-13$ \\
\hline & $u_{x_{2}}\left(P_{1}\right)$ & $1.7719 \mathrm{e}-06$ & $3.7526 \mathrm{e}-14$ & $5.3346 \mathrm{e}-14$ \\
\hline & $u_{x_{2}}\left(P_{2}\right)$ & $4.6493 \mathrm{e}-12$ & $2.3209 \mathrm{e}-13$ & $9.8088 \mathrm{e}-14$ \\
\hline & $u_{x_{1} x_{1}}\left(P_{1}\right)$ & $1.8116 \mathrm{e}-04$ & $1.1985 \mathrm{e}-11$ & $3.0287 \mathrm{e}-12$ \\
\hline & $u_{x_{1} x_{1}}\left(P_{2}\right)$ & $1.0911 \mathrm{e}-11$ & $9.3148 \mathrm{e}-14$ & 7.6272e-14 \\
\hline & $u_{x_{1} x_{2}}\left(P_{1}\right)$ & $2.7358 \mathrm{e}-04$ & $5.6236 \mathrm{e}-11$ & $2.0127 \mathrm{e}-12$ \\
\hline & $u_{x_{1} x_{2}}\left(P_{2}\right)$ & $2.3542 \mathrm{e}-11$ & $1.0200 \mathrm{e}-12$ & $5.4058 \mathrm{e}-13$ \\
\hline
\end{tabular}

\begin{tabular}{|c|ccccc|}
\hline & $u\left(P_{j}\right)$ & $u_{x_{1}}\left(P_{j}\right)$ & $u_{x_{2}}\left(P_{j}\right)$ & $u_{x_{1} x_{1}}\left(P_{j}\right)$ & $u_{x_{1} x_{2}}\left(P_{j}\right)$ \\
\hline$P_{1}$ & $8.0449 \mathrm{e}-02$ & $8.2401 \mathrm{e}-01$ & $3.4132 \mathrm{e}-01$ & $1.1653 \mathrm{e}+00$ & $-4.8270 \mathrm{e}-01$ \\
$P_{2}$ & $1.7611 \mathrm{e}+00$ & $1.8618 \mathrm{e}+00$ & $-3.0213 \mathrm{e}-01$ & $5.8875 \mathrm{e}-01$ & $9.5541 \mathrm{e}-02$ \\
\hline
\end{tabular}


Example 3.2 (Boomerang Domain). The boundary of the Boomerang domain is parametrized by

$$
x(t)=(-(2 / 3) \sin (3 t / 2), \beta \sin t) \quad, \quad \beta=\tan (\pi /(2 \alpha)),
$$

for some fixed $1 / 2<\alpha<1$, and $t \in[0,2 \pi)$. In this case choosing $\alpha=2 / 3$ gives a boundary length of $|\partial \Omega| \approx 6.038382737179698567469163685137$, correct to all digits shown. For $n=32,64,128$ and fixed Kress quadrature parameter $p=7$, we report the maximal errors in $W^{\prime}$, as well as pointwise errors in the approximation of $u$ and its derivatives at two test points in $\Omega$, in Table 5 . Increasing the interpolation parameter to $\zeta=4$ only yielded significant improvement of the approximation of the second derivatives at one of the points, $P_{2}$, when $n=32$. The errors in this case were $2.6027 \mathrm{e}-07$ and $1.1944 \mathrm{e}-07$ for $u_{x_{1} x_{1}}\left(P_{2}\right)$ and $u_{x_{1} x_{2}}\left(P_{2}\right)$, respectively.

3.2. Domains with Multiple Corners. We now consider domains with $m$ corners. Proceeding counterclockwise along $\partial \Omega$, label the successive corner points $z_{0}, \ldots, z_{m-1} \partial \Omega$, taking $z_{m}=z_{0}$ for convenience. Let $\partial \Omega_{k}$ be the arc (edge) of $\partial \Omega$ having endpoints $z_{k}$ and $z_{k+1}$. For the $k$ th edge, we introduce the parameterization $x_{k}:[0,2 \pi] \rightarrow \partial \Omega_{k}$. The overall parameterization $x:[0,2 m \pi] \rightarrow \partial \Omega$, taken counterclockwise, may then be described by

$$
x(t)=x_{k}(t-2 k \pi) \text { when } t \in[2 k \pi, 2(k+1) \pi], \text { for } k=0, \ldots, m-1 .
$$

The remaining modification to make in (26) is to choose $z=z(x)$ to be to the nearest corner point for each $x \in \partial \Omega$. More specifically, if $x$ is a corner point, then $z(x)=x$; otherwise, if $x \in \partial \Omega_{k}, z(x)$ is the nearer of

TABLE 5. Boomerang: Errors in approximating $W^{\prime}(\tau)$ on $\partial \Omega$, and the values of $u$ and its first and second partial derivatives at two points, $P_{1}=(0,1 / 5)$ and $P_{2}=(1 / 5,-2 / 5)$, in $\Omega$. Below this are the exact values of $u$ and its first and second partial derivatives at these points.

\begin{tabular}{|c|c|ccc|}
\hline & & $n=32$ & $n=64$ & $n=128$ \\
\hline & $W^{\prime}(\tau)$ & $9.4803 \mathrm{e}-05$ & $7.0329 \mathrm{e}-06$ & $3.7774 \mathrm{e}-07$ \\
\hline \multirow{5}{*}{$\zeta=1$} & $u\left(P_{1}\right)$ & $2.5641 \mathrm{e}-06$ & $4.7332 \mathrm{e}-11$ & $9.2051 \mathrm{e}-13$ \\
& $u\left(P_{2}\right)$ & $1.1966 \mathrm{e}-04$ & $4.6305 \mathrm{e}-08$ & $1.6187 \mathrm{e}-13$ \\
& $u_{x_{1}}\left(P_{1}\right)$ & $1.7859 \mathrm{e}-04$ & $1.1043 \mathrm{e}-09$ & $5.4297 \mathrm{e}-12$ \\
& $u_{x_{1}}\left(P_{2}\right)$ & $1.3322 \mathrm{e}-03$ & $3.8414 \mathrm{e}-06$ & $3.0368 \mathrm{e}-12$ \\
& $u_{x_{2}}\left(P_{1}\right)$ & $3.3131 \mathrm{e}-05$ & $2.2396 \mathrm{e}-09$ & $1.4309 \mathrm{e}-12$ \\
& $u_{x_{2}}\left(P_{2}\right)$ & $6.2385 \mathrm{e}-03$ & $9.5505 \mathrm{e}-07$ & $2.2362 \mathrm{e}-12$ \\
& $u_{x_{1} x_{1}}\left(P_{1}\right)$ & $1.2630 \mathrm{e}-02$ & $3.8802 \mathrm{e}-08$ & $5.8864 \mathrm{e}-12$ \\
& $u_{x_{1} x_{1}}\left(P_{2}\right)$ & $1.9192 \mathrm{e}-01$ & $1.0969 \mathrm{e}-04$ & $3.1104 \mathrm{e}-11$ \\
& $u_{x_{1} x_{2}}\left(P_{1}\right)$ & $5.2302 \mathrm{e}-03$ & $3.9446 \mathrm{e}-07$ & $2.5246 \mathrm{e}-11$ \\
& $u_{x_{1} x_{2}}\left(P_{2}\right)$ & $9.6834 \mathrm{e}-02$ & $2.4007 \mathrm{e}-04$ & $9.4683 \mathrm{e}-11$ \\
\hline \multirow{5}{*}{$\zeta=2$} & $u_{1}\left(P_{1}\right)$ & $1.0626 \mathrm{e}-07$ & $5.5637 \mathrm{e}-11$ & $9.2112 \mathrm{e}-13$ \\
& $u\left(P_{2}\right)$ & $8.6896 \mathrm{e}-08$ & $2.7288 \mathrm{e}-11$ & $1.6676 \mathrm{e}-13$ \\
& $u_{x_{1}}\left(P_{1}\right)$ & $5.7167 \mathrm{e}-08$ & $3.1948 \mathrm{e}-10$ & $5.4287 \mathrm{e}-12$ \\
& $u_{x_{1}}\left(P_{2}\right)$ & $3.6962 \mathrm{e}-06$ & $3.5486 \mathrm{e}-11$ & $2.2881 \mathrm{e}-12$ \\
& $u_{x_{2}}\left(P_{1}\right)$ & $2.8798 \mathrm{e}-07$ & $2.6696 \mathrm{e}-10$ & $1.4291 \mathrm{e}-12$ \\
& $u_{x_{2}}\left(P_{2}\right)$ & $7.8038 \mathrm{e}-07$ & $1.3160 \mathrm{e}-10$ & $1.9919 \mathrm{e}-12$ \\
& $u_{x_{1} x_{1}}\left(P_{1}\right)$ & $1.4949 \mathrm{e}-06$ & $1.9932 \mathrm{e}-09$ & $5.8825 \mathrm{e}-12$ \\
& $u_{x_{1} x_{1}}\left(P_{2}\right)$ & $1.0943 \mathrm{e}-04$ & $4.7297 \mathrm{e}-10$ & $5.1005 \mathrm{e}-12$ \\
& $u_{x_{1} x_{2}}\left(P_{1}\right)$ & $2.2716 \mathrm{e}-06$ & $3.1653 \mathrm{e}-09$ & $2.5230 \mathrm{e}-11$ \\
& $u_{x_{1} x_{2}}\left(P_{2}\right)$ & $2.4019 \mathrm{e}-04$ & $3.1828 \mathrm{e}-11$ & $3.1751 \mathrm{e}-12$ \\
\hline
\end{tabular}

\begin{tabular}{|c|ccccc|}
\hline & $u\left(P_{j}\right)$ & $u_{x_{1}}\left(P_{j}\right)$ & $u_{x_{2}}\left(P_{j}\right)$ & $u_{x_{1} x_{1}}\left(P_{j}\right)$ & $u_{x_{1} x_{2}}\left(P_{j}\right)$ \\
\hline$P_{1}$ & $1.7100 \mathrm{e}-01$ & $9.8726 \mathrm{e}-01$ & $5.6999 \mathrm{e}-01$ & $9.4999 \mathrm{e}-01$ & $-1.6454 \mathrm{e}+00$ \\
$P_{2}$ & $4.3261 \mathrm{e}-01$ & $8.1308 \mathrm{e}-01$ & $-3.1447 \mathrm{e}-01$ & $-6.1376 \mathrm{e}-02$ & $6.4688 \mathrm{e}-01$ \\
\hline
\end{tabular}




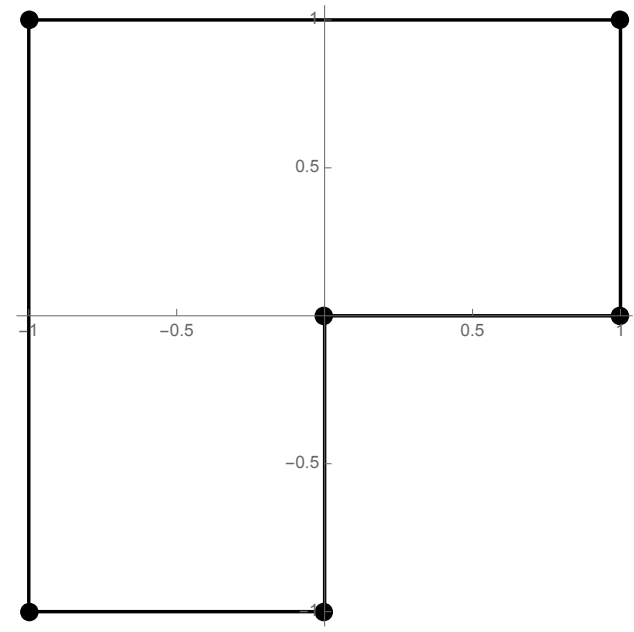

Figure 5. Polygon: The hexagonal domain $\Omega$ used in Example 3.3.

the two endpoints $z_{k}$ and $z_{k+1}$. In light of these modifications, the parameterized version of (26) is

$$
|\partial \Omega| c(t)+\frac{V(t)-c(t)}{2}+\int_{0}^{2 m \pi} K(t, s)(V(s)-c(t)) d s=\int_{0}^{2 m \pi}\left(L_{1}(t, s)+L_{2}(t, s)\right) G(s) d s
$$

where $c(t)=v(z(x(t)))$. We again approximate these integrals by employing a combination of Kress and Martensen quadratures, as in the single corner case, on each edge. Interpolation of the computed data is done over the whole boundary, not over the individual edges.

Example 3.3 (Polygon Domain). Let $\Omega$ be the non-convex hexagon having vertices

$$
\{(0,0),(1,0),(1,1),(-1,1),(-1,-1),(0,-1)\},
$$

listed in counter-clockwise order-see Figure 5. The (initial) parameterization of the boundary is given by (33), with

$$
x_{k}(t)=\frac{2 \pi-t}{2 \pi} z_{k}+\frac{t}{2 \pi} z_{k+1}
$$

We consider the harmonic conjugate pair

$$
u=r^{\alpha} \sin (\alpha \theta) \quad, \quad v=-r^{\alpha} \cos (\alpha \theta),
$$

where $\alpha=2 / 3$. Note that the average value of $v$ on $\partial \Omega$ is 0 . The singular behavior of $u($ and $v)$ near the re-entrant corner is typical for the interior angle $\pi / \alpha=3 \pi / 2$. As with the previous examples, we use the Kress quadrature parameter $p=7$, and vary both the level of discretization $n$ and the level of interpolation $\zeta$. The high-order convergence with respect to $n$ is again observed in Table 6.

Example 3.4 (Polygon Domain Revisited). Let $\Omega$ be as in the previous example. For $0 \leq k \leq 5$, let $f_{k} \in C(\partial \Omega)$ be the piecewise linear function defined by the relations $f_{k}\left(z_{j}\right)=\delta_{j k}$. Taking $f_{k}$ to be the Dirichlet data for $u$, the corresponding Neumann data for $v$ is piecewise constant. This example is motivated by BEM-FEM discretizations, as discussed in the Introduction. The analytical solutions are unknown, but we numerically estimate $u,|\nabla u|$, and $\partial u / \partial \mathbf{n}$ and report the results in Figure 6 , for the cases $k=0,1,2,3$. The cases $k=4,5$ can be obtained by using the symmetry of the domain. We use $n=32, p=7$, and $\zeta=8$, and evaluate $u$ and $|\nabla u|$ on a uniform grid with spacing $1 / 64$ to obtain contour plots. Using $n=128$ and $p=7$ ( $\zeta$ is irrelevant in this case), the normal derivative is approximated as suggested in (32), and plotted versus the parameter $t$ over $[0,12 \pi]$. We see that $\partial u / \partial \mathbf{n}$ is unbounded at the non-convex corner $(t=0,12 \pi)$ in each case, as is typically expected, and has jump discontinuities at some of the remaining corners. Increasing the parameters made no difference in these plots. 
TABle 6. Polygon: Errors in approximating $W^{\prime}(\tau)$ on $\partial \Omega$, and the values of $u$ and its first and second partial derivatives at two points, $P_{1}=(1 / 4,9 / 10)$ and $P_{2}=(-1 / 20,-1 / 20)$, in $\Omega$. Below this are the exact values of $u$ and its first and second partial derivatives at these points.

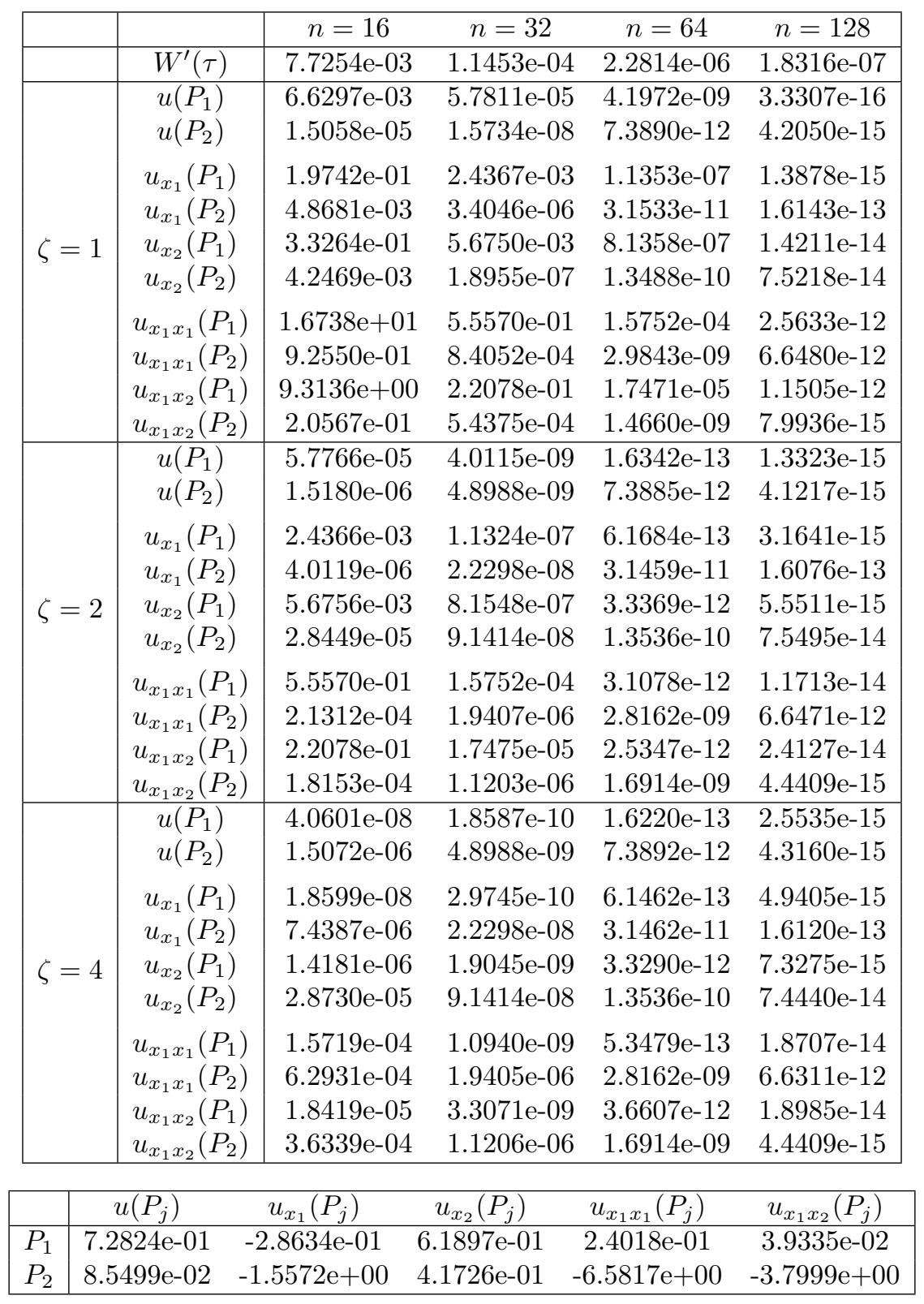

\section{REFERENCES}

[1] O. P. Bruno, J. S. Ovall, and C. Turc. A high-order integral algorithm for highly singular PDE solutions in Lipschitz domains. Computing, 48(3):149-181, 2009.

[2] D. Copeland, U. Langer, and D. Pusch. From the boundary element domain decomposition methods to local Trefftz finite element methods on polyhedral meshes. In Domain decomposition methods in science and engineering XVIII, volume 70 of Lect. Notes Comput. Sci. Eng., pages 315-322. Springer, Berlin, 2009.

[3] P. Grisvard. Elliptic problems in nonsmooth domains, volume 24 of Monographs and Studies in Mathematics. Pitman (Advanced Publishing Program), Boston, MA, 1985.

[4] P. Henrici. Fast Fourier methods in computational complex analysis. SIAM Rev., 21(4):481-527, 1979. 

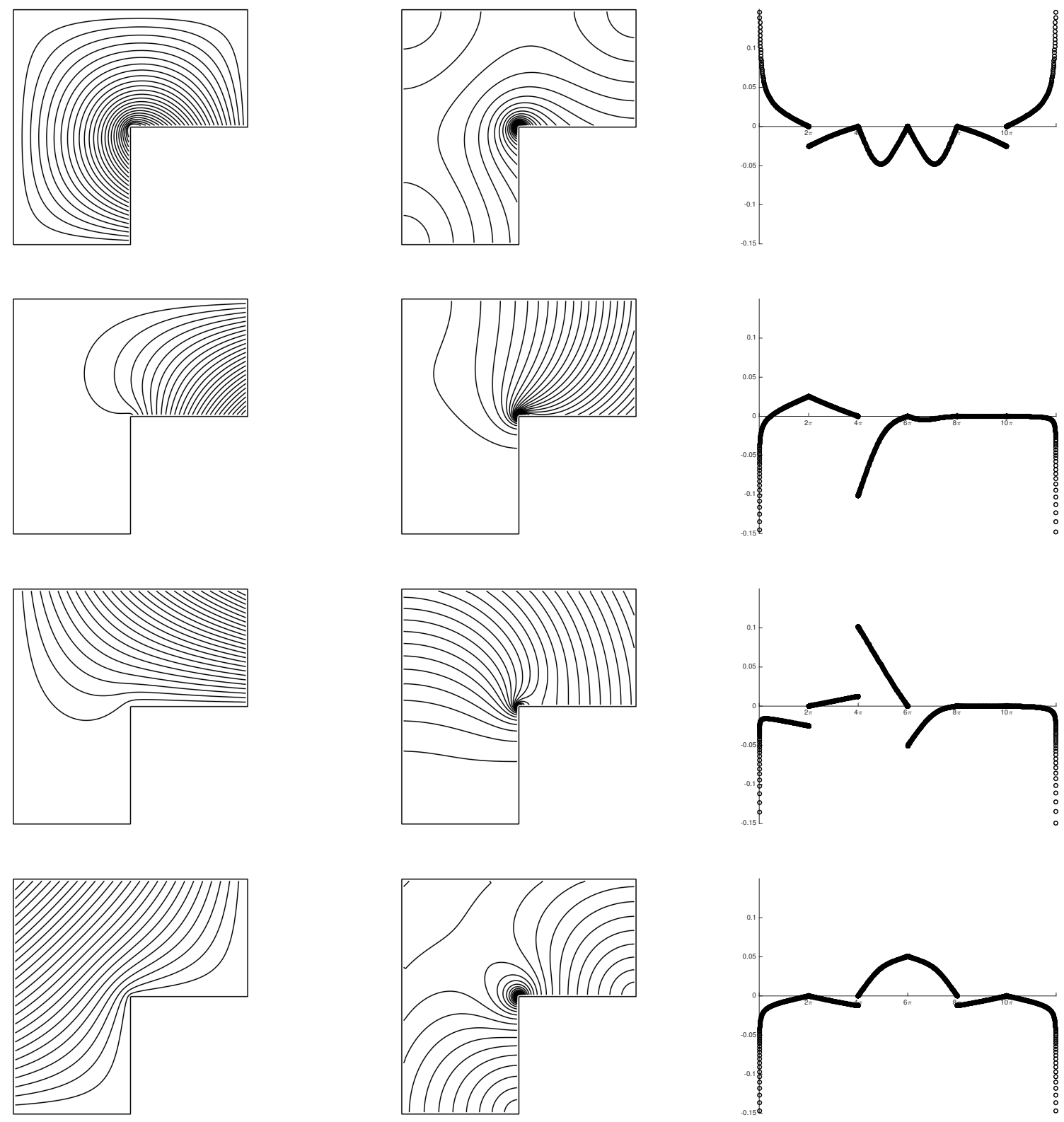

Figure 6. Polygon: Contour plots of $u$ (left column) and $|\nabla u|$ (center column) for piecewise linear data considered in Example 3.4. The normal derivative $\partial u / \partial \mathbf{n}$ is plotted in the right column. Rows correspond to $k=0,1,2,3$, respectively.

[5] C. Hofreither. $L_{2}$ error estimates for a nonstandard finite element method on polyhedral meshes. J. Numer. Math., 19(1):2739, 2011.

[6] C. Hofreither, U. Langer, and C. Pechstein. Analysis of a non-standard finite element method based on boundary integral operators. Electron. Trans. Numer. Anal., 37:413-436, 2010.

[7] R. Kress. A Nyström method for boundary integral equations in domains with corners. Numer. Math., 58(2):145-161, 1990.

[8] R. Kress. Linear integral equations, volume 82 of Applied Mathematical Sciences. Springer, New York, third edition, 2014.

[9] R. Kussmaul. Ein numerisches Verfahren zur Lösung des Neumannschen Neumannschen Aussenraumproblems für die Helmholtzsche Schwingungsgleichung. Computing (Arch. Elektron. Rechnen), 4:246-273, 1969.

[10] E. Martensen. Über eine Methode zum räumlichen Neumannschen Problem mit einer Anwendung für torusartige Berandungen. Acta Math., 109:75-135, 1963. 
[11] E. J. Nyström. Über Die Praktische Aufösung von Integralgleichungen mit Anwendungen auf Randwertaufgaben der Potentialtheorie. Soc. Sci. Fenn. Comment. Phys.-Math., 4(15):1-52, 1928.

[12] E. J. Nyström. Über Die Praktische Aufösung von Integralgleichungen mit Anwendungen auf Randwertaufgaben. Acta Math., 54(1):185-204, 1930.

[13] S. Rjasanow and S. Weißer. Higher order BEM-based FEM on polygonal meshes. SIAM J. Numer. Anal., 50(5):2357-2378, 2012.

[14] S. Rjasanow and S. Weißer. FEM with Trefftz trial functions on polyhedral elements. J. Comput. Appl. Math., 263:202-217, 2014.

[15] Y. Saad and M. H. Schultz. GMRES: a generalized minimal residual algorithm for solving nonsymmetric linear systems. SIAM J. Sci. Statist. Comput., 7(3):856-869, 1986.

[16] L. Trefethen and J. Weideman. The exponentially convergent trapezoidal rule. SIAM Review, 56(3):385-458, 2014.

[17] S. Weißer. Residual error estimate for BEM-based FEM on polygonal meshes. Numer. Math., 118(4):765-788, 2011.

[18] S. Weißer. Arbitrary order Trefftz-like basis functions on polygonal meshes and realization in BEM-based FEM. Comput. Math. Appl., 67(7):1390-1406, 2014.

[19] S. Weißer. Residual based error estimate and quasi-interpolation on polygonal meshes for high order bem-based $\{$ FEM $\}$. Computers \& Mathematics with Applications, 73(2):187 - 202, 2017.

[20] N. M. Wigley. Asymptotic expansions at a corner of solutions of mixed boundary value problems. J. Math. Mech., 13:549$576,1964$.

[21] S. S. Zargaryan and V. G. Maz'ya. The asymptotic form of the solutions of integral equations of potential theory in the neighbourhood of the corner points of a contour. Prikl. Mat. Mekh., 48(1):169-174, 1984.

Jeffrey S. Ovall, Fariborz Maseeh Department of Mathematics and Statistics, Portland State University, Portland, OR 97201

E-mail address: jovall@pdx.edu

Samuel Reynolds, Fariborz Maseeh Department of Mathematics and Statistics, Portland State University, Portland, OR 97201

E-mail address: ser6@pdx.edu 